\title{
Size Effects on Surface Elastic Waves in a Semi-Infinite Medium with Atomic Defect Generation
}

\author{
F. Mirzade \\ Institute on Laser and Information Technologies, Russian Academy of Sciences, Moscow 140700, Russia \\ Correspondence should be addressed to F. Mirzade; fmirzade@rambler.ru
}

Received 3 May 2013; Accepted 10 October 2013

Academic Editor: Michael C. Tringides

Copyright (C) 2013 F. Mirzade. This is an open access article distributed under the Creative Commons Attribution License, which permits unrestricted use, distribution, and reproduction in any medium, provided the original work is properly cited.

The paper investigates small-scale effects on the Rayleigh-type surface wave propagation in an isotopic elastic half-space upon laser irradiation. Based on Eringen's theory of nonlocal continuum mechanics, the basic equations of wave motion and laser-induced atomic defect dynamics are derived. Dispersion equation that governs the Rayleigh surface waves in the considered medium is derived and analyzed. Explicit expressions for phase velocity and attenuation (amplification) coefficients which characterize surface waves are obtained. It is shown that if the generation rate is above the critical value, due to concentration-elastic instability, nanometer sized ordered concentration-strain structures on the surface or volume of solids arise. The spatial scale of these structures is proportional to the characteristic length of defect-atom interaction and increases with the increase of the temperature of the medium. The critical value of the pump parameter is directly proportional to recombination rate and inversely proportional to deformational potentials of defects.

\section{Introduction}

During the last decades elastic wave propagation in solids (metals and semiconductors) with nonequilibrium atomic defects has received a lot of attention [1-4]. Various types of lattice defects (vacancies and interstitials), produced from the lattice site atoms due to intense external energy fluxes (laser and corpuscular radiations), introduce a significant strain of the medium as a result of the difference between the radii of lattice atoms and defects [5] and play an important role in surface modification of solids exposed to laser radiation $[1,5,6]$. The formation of atomic defects may occur also in a number of other technologies processes: in the laser fast recrystallization, laser annealing, multipulse laser etching, pulsed laser-assisted thin-film deposition, and so forth. Strains in an elastic wave cause a strain-induced drift of defects, whereas the strains and a variation in the temperature in the wave modulate the rates of generation and recombination of defects of the thermal-fluctuation origin (via variations in the energies of the defect formation and migration) $[5,6]$.

Several mathematical models have been considered to study the self-organization of two-dimensional (2D) ordered microscale concentration-strain (CS) structures (at high concentrations of atomic defects) on the surface of the solid halfspace $[7,8]$ and in an isotropic solid elastic layer $[9,10]$ under the action of laser irradiation. In these studies, attention was also focused on the study of an influence of the straininduced diffusion, generation, and annihilation of defects on the propagation characteristics of strain waves. In both [1$4]$ and [7-10] the analysis was based on coupled evolution equations for the atomic defect density fields and the classical local elasticity equations for the elastic displacement of the medium.

Classical continuum theory is characterized by the local character of stress (stress at a point depends only on the strain at that point) and does not contain internal length scale. The absence of the length scale creates several discrepancies in the predictions of mechanical responses, for example, nondispersive wave behavior (constant wave velocity, independent of frequency). For example, according to the classical elasticity, Rayleigh waves propagating on the surface of a semi-infinite isotropic elastic space are not dispersive at any frequency, whereas experiments and the atomic theory of lattice predict otherwise. 
Limitations of the classical elasticity theory are also demonstrated in the study of the formation of coupled straindefect nanometer sized ordered structures (short-range CS structures) on the surface of laser-irradiated solids and mechanical behavior of microstructured materials, because their behavior is characterized by nonlocal stresses and the existence of an internal length scale [11, 12]. The accurate analysis of dynamic behaviors of these structures cannot be correctly described by classical local elasticity theory. Conducting experiments with nanoscale size materials is found to be difficult and expensive. Therefore, development of appropriate nonlocal elasticity mathematical models for nanostructure formation and nanomaterials is of great importance.

In contrast to local approach of zero-range internal interactions, the nonlocal elasticity theory, originated and developed in the last four decades, postulates that the stress at a point $x$ in a body depends not only on the strain at point $x$ but also on those at all other points of the body. Various nonlocal theories of linear elasticity have been proposed to describe the scale effects on the characteristics of the vibration and elastic wave propagation in the abovementioned submicro- or nanosized structures. The basic constitutive equations and governing equations of linear nonlocal elasticity were derived by Eringen [11] and Kunin [12]. Later the nonlocal theories have been applied for the analysis of micro- and nanoscale plate-like structures, in which the small-scale effects become significant. In [13] Eringen considered vibration behavior of a nanoplate by using linear theory of nonlocal continuum mechanics. By using [11], Reddy [14] reformulated the classical and shear deformation beam and plate theories. The nonlocal scale influences on the wave dispersion properties of the nanoplates are discussed in detail in [15]. Vibration characteristics of nanoplates, based on three-dimensional theory of elasticity employing nonlocal continuum mechanics, have been discussed in [15]. A review of some other applications of nonlocal elasticity theories for nanostructures can be found in [16]. Propagation of longitudinal elastic and thermoelastic waves in an isotropic, homogeneous infinite medium with long-rang interactions has been studied by Nowinski $[17,18]$. Other advances have been made by the application of nonlocal elasticity to such fields as the solid defects $[19,20]$ and fracture mechanics [21].

The present paper is concerned with the nonlocal elasticity theory of laser-excited solid half-space with mobile atomic point defects. We summarise the theory formulated in [8] to analyze the effects of nonlocal atom-atom and atom-defect interactions on the surface wave propagation in solids with defect generation. The changes in defect concentration are controlled by the (i) generation of defects by laser irradiation, (ii) their diffusion, and (iii) strain-induced diffusion. Dispersion equation that governs the propagation of elasticconcentration waves has been derived by solving a system of coupled partial differential equations. Some limiting cases of the dispersion equations are considered. For a small value of coupled parameter obvious expressions for the phase velocity and the attenuation (amplification) factor of surface elasticconcentration waves are determined. It is found that both phase velocity and attenuation coefficient are to be influenced by the presence of defect generation. We also obtain that at certain conditions concentration-elastic instabilities with the formation of regular nanosized structures in a system of atomic defects on the surface of the solids can be developed. We demonstrate that due to the nonlocal character of the defect-atom and atom-atom interactions the dispersive curve of the instability has two maxima. As a result, size distribution functions of the surface nanoscale nonhomogeneities having two maxima can be formed. The results of some earlier works are also deduced from the present formulation. To our knowledge, the problem in its present form was not investigated before.

\section{Governing Equations}

In this section we give basic $2 \mathrm{D}$ equations governing the defect density and elastic displacement fields based on the nonlocal constitutive relations of the elasticity theory. Consider an isotopic elastic semi-infinite medium occupying a region $-\infty<x<\infty, 0<z<\infty$ in a rectangular Cartesian coordinate system $O x, y, z$, where the origin $O$ is situated at any point on the plane boundary and $\mathrm{O} z$ points vertically downwards, that is, towards the bulk of the medium. The surface $z=0$ is supposed to be free from stresses. Let a plane elastic wave propagate along the $x$-axis. Denoting $u$ and $v$ as the nonzero components of the displacement vector $\vec{u}$, we set $u=u(x, z, t), v=v(x, z, t)$, and $w=0$ and consequently write for the strain tensor $\left(e_{i j}\right)$ :

$$
e_{x x}=\frac{\partial u}{\partial x}, \quad e_{z z}=\frac{\partial v}{\partial z}, \quad 2 e_{x z}=\left(\frac{\partial u}{\partial z}+\frac{\partial v}{\partial x}\right) .
$$

Let us assume that an external energy flux (e.g., laser radiation) creates mobile atomic defects in a surface layer. The corresponding defect density profile results in a force that may induce strain field in medium. Let $n_{i}(x, z, t)$ be the density of these defects of the $j$ th-type $(i=V$ for vacancies ( $V$-defects) and $j=I$ for interstitials ( $I$-defects $)$ ). We limit our consideration to the case of only one type of atomic defects (for definiteness, $V$-type defects).

The concentration field of atomic defects is dependent on temperature of the medium. One thus needs to know how the laser irradiation affects the local temperature of the surface at the laser spot. We will consider here situations where the laser irradiation only heats the solid (the light energy absorbed by the medium is transformed into heat) and that an equilibrium between laser radiation and the temperature field $(T)$ is reached on time scales much shorter than the characteristic time scale of defect density evolution. Typically, the time scale for equilibration between photon absorption and defect generation is on the order of picoseconds, while that for defect diffusion is of the order of microseconds. We also assume that the contribution of thermal strains to deformation fields is negligible compared to lattice dilatation due to atomic defects and the phase changes and chemical reactions in the medium are absent.

In this paper, we will consider the problem of the wave propagation in an elastic solid irradiated over a large area by $\mathrm{CW}$ or pulsed lasers. Furthermore, we will assume that 
the temperature field has reached its equilibrium value. Its evolution is sufficiently slow compared to atomic defect generation and can be considered as quasistationary. The solution of the heat conduction equation for this case is given by Duley [22].

Taking into account the defect generation, the constitutive equations of an isotropic nonlocal elastic solid are

$$
\begin{aligned}
\sigma_{i k}(\mathbf{x}, t)= & \int_{\Omega} \alpha\left(\left|\mathbf{x}^{\prime}-\mathbf{x}\right|\right) s_{i k}\left(\mathbf{x}^{\prime}, t\right) d \Omega\left(\mathbf{x}^{\prime}\right) \\
& -\int_{\Omega} \beta\left(\left|\mathbf{x}^{\prime}-\mathbf{x}\right|\right) n\left(\mathbf{x}^{\prime}, t\right) \delta_{i k} d \Omega\left(\mathbf{x}^{\prime}\right), \\
s_{i k}\left(\mathbf{x}^{\prime}, t\right)= & \lambda_{0} e\left(\mathbf{x}^{\prime}, t\right) \delta_{i k}+2 \mu_{0} e_{i k}\left(\mathbf{x}^{\prime}, t\right),
\end{aligned}
$$

where $\sigma_{i k}$ is the nonlocal stress tensor, $s_{i k}$ is the classical stress tensor, $\lambda_{0}$ and $\mu_{0}$ are Lamé's constants [23], $e_{i k}=$ $\left(u_{i, k}+u_{k, i}\right) / 2$ is the strain tensor, $e=u_{k, k}$ is the dilatation, and $d \Omega\left(\mathbf{x}^{\prime}\right)=d x_{1}^{\prime} d x_{2}^{\prime}$. The functions $\alpha$ and $\beta$ are known as atom-atom (short-range) and atom-defect (long-range) interaction kernels or moduli of nonlocality, which decay smoothly with distance. They reflect the influence of an independent constitutive variable at a point $\mathbf{x}^{\prime}$ on a dependent constitutive variable at $x$. Nonlocal moduli are physical properties of materials like other physical constants and need to be determined experimentally.

The basic difference between classical and nonlocal elasticity is in the presence of the volume integrals in (2) which indicates that the stress at $(\mathbf{x}, t)$ depends on the strain and defect density at all other points $\left\{\mathbf{x}^{\prime}\right\}$ of the body, at time $t$. This signifies that the distant neighbors of a point $\mathbf{x}$ have a role to play in the propagation of waves.

In absence of body forces the equations of motion and the equations of defect density dynamics have the following form:

$$
\begin{gathered}
\frac{\rho \partial^{2} u_{i}}{\partial t^{2}}+\nabla_{k} \sigma_{i k}=0 \\
\frac{\partial n}{\partial t}=G+D \nabla^{2} n-\frac{D}{k_{B} T} \nabla \cdot(n \mathbf{f})-n \tau^{-1}
\end{gathered}
$$

where $\rho$ is the density of the medium, $G=G_{0} \exp \left(-w_{g} / k_{B} T\right)$ is the thermal-fluctuation generation rate of atomic defects at sites $\left(G_{0}\right.$ is the constant and $k_{B}$ is the Boltzmann constant), and $\tau=\tau_{0} \exp \left(w_{m} / k_{B} T\right)$ is the relaxation time of defects in the absence; $w_{g}$ and $w_{m}$ are the formation and migration energies for the defects in crystals.

The first term in the right-hand side of (4) takes into account laser-induced generation of defects, the second term represents diffusion with a coefficient $D$, the third term corresponds to the drift of defects under the influence of the force $\mathbf{f}=-\nabla U_{\text {int }}$ resulting from the nonlocal interaction of defects with an inhomogeneous strain field, and the fourth term describes the rate of their disappearance due to recombination processes. It is assumed that the generation rate $(G)$ is spatially uniform.
The expression for interaction energy $U_{\text {int }}$ of a defect with the strain field $e_{m m}$ in a nonlocal elastic medium is given by the formula

$$
U_{\mathrm{int}}=-\int_{\Omega} \beta\left(\left|\mathbf{x}^{\prime}-\mathbf{x}\right|\right) e\left(\mathbf{x}^{\prime}\right) d \Omega\left(\mathbf{x}^{\prime}\right)
$$

In the limit, when the kernel functions $\alpha$ and $\beta$ become Dirac-delta functions, (2) reduces to the classical constitutive equations of local elasticity theory.

Thus the final dynamic field equations obtained are integrodifferential equations for the functions $u(x, t)$ and $n(x, t)$. It seems to be obvious that a rigorous solution of such equations encounters serious but not insurmountable mathematical difficulties. However, these equations can be reduced to the partial differential forms under certain conditions with physical admissible kernels. We consider that the long-range internal influences of particles of the body are rather rapidly with increasing distance from the particle. Thus the 2D-kernel functions that characterize the nonlocal interaction in the $z$-direction may be approximated in terms of delta-like functions as

$$
\begin{aligned}
& \alpha=\alpha\left(\left|x^{\prime}-x\right|,\left|z^{\prime}-z\right|\right)=\alpha\left(\left|x^{\prime}-x\right|\right) \delta_{n}\left(z^{\prime}-z\right), \\
& \beta=\beta\left(\left|x^{\prime}-x\right|,\left|z^{\prime}-z\right|\right)=\beta\left(\left|x^{\prime}-x\right|\right) \delta_{n}\left(z^{\prime}-z\right) .
\end{aligned}
$$

Besides the nonlocal kernels $\alpha$ and $\beta$ are symmetric with respect to the interchange of the arguments $\xi=\mathbf{x}^{\prime}-\mathbf{x}$. Then expanding in (3) the functions $e(\mathbf{x}+\xi)$ and $n(\mathbf{x}+\xi)$ in Taylor series around $\boldsymbol{\xi}=0$ and neglecting the terms higher than second-order gradients, we can obtain the following expression for the nonlocal constitutive relation with strain and defect concentration gradients:

$$
\begin{aligned}
\sigma_{i k}(\mathbf{x})= & \left(1+g^{2} \nabla^{2}\right)\left[\lambda_{0} \delta_{i k} e(\mathbf{x})+2 \mu_{0} e_{i k}(\mathbf{x})\right] \\
& -\left(1+h^{2} \nabla^{2}\right) \beta_{0} \delta_{i k} n(\mathbf{x})
\end{aligned}
$$

where $h$ and $g$ are the intrinsic characteristic length scale parameters, characterizing the defect-atom and atom-atom interactions, respectively; $\nabla^{2}=\partial^{2} / \partial x^{2} ; \beta_{0}=K \Omega_{d}$ is deformation potential of the defect $\left(\Omega_{d}\right.$ is the change of the volume of the medium under formation of a single defect and $K$ is the bulk modulus). The gradient coefficients $g$ and $h$ are dependent on the lattice or atomic chain model used and the interatomic potentials assumed. The values of these coefficients are taken to be order of interatomic distance. For the interaction energy $U_{\text {int }}$ we have from (5) $U_{\text {int }}=-(1+$ $\left.h^{2} \nabla^{2}\right) \beta_{0} e$.

To obtain the nonzero stress components in terms of the displacement components we substitute (1) into (7):

$$
\begin{aligned}
\sigma_{x x}= & \left(1+g^{2} \nabla^{2}\right)\left[\left(\lambda_{0}+2 \mu_{0}\right) \frac{\partial u}{\partial x}+\lambda_{0} \frac{\partial v}{\partial z}\right] \\
& -\left(1+h^{2} \nabla^{2}\right) \beta_{0} n,
\end{aligned}
$$




$$
\begin{array}{r}
\sigma_{z z}=\left(1+g^{2} \nabla^{2}\right)\left[\left(\lambda_{0}+2 \mu_{0}\right) \frac{\partial v}{\partial z}+\lambda_{0} \frac{\partial u}{\partial x}\right] \\
-\left(1+h^{2} \nabla^{2}\right) \beta_{0} n, \\
\sigma_{x z}=\left(1+g^{2} \nabla^{2}\right) \mu_{0}\left(\frac{\partial u}{\partial z}+\frac{\partial v}{\partial x}\right) .
\end{array}
$$

Then from (4), two nonzero equations of motion can be obtained:

$$
\begin{aligned}
& \rho \frac{\partial^{2} u}{\partial t^{2}}=\frac{\partial \sigma_{x x}}{\partial x}+\frac{\partial \sigma_{x z}}{\partial z}, \\
& \rho \frac{\partial^{2} v}{\partial t^{2}}=\frac{\partial \sigma_{z x}}{\partial x}+\frac{\partial \sigma_{z z}}{\partial z} .
\end{aligned}
$$

Suppose that the stress components and defect density field satisfy the boundary conditions

$$
\begin{gathered}
\sigma_{z z}=\sigma_{z x}=0, \quad \frac{\partial n}{\partial z}=0, \quad \text { for } z=0 ; \\
u, v, n \longrightarrow 0 \quad \text { as } z \longrightarrow \infty .
\end{gathered}
$$

We can express the defect density field as $n=n_{0}+$ $n_{1}\left(n_{0}=G \tau\right.$ is a spatially homogeneous solution; $n_{1}$ is small nonhomogeneous perturbations). Inserting in (4) the nonlinear terms, we get the linearised equation as

$$
\begin{aligned}
\frac{\partial n_{1}}{\partial t} & +\frac{n_{1}}{\tau}-D\left(\frac{\partial^{2} n_{1}}{\partial x^{2}}+\frac{\partial^{2} n_{1}}{\partial z^{2}}\right) \\
= & -\frac{n_{0} \beta_{0} D}{k_{B} T}\left[\frac{\partial^{2} e}{\partial x^{2}}+\frac{\partial^{2} e}{\partial z^{2}}+g^{2}\left(\frac{\partial^{4} e}{\partial x^{4}}+\frac{\partial^{2} e}{\partial x^{2} \partial z^{2}}\right)\right]
\end{aligned}
$$

The system of (9) and (11) is closely coupled. $\partial^{2} \vec{u} / \partial t^{2}$ in (9) depends on the defect density field $\left(n_{1}\right)$ and $\partial n_{1} / \partial t$ in (11) depends on elastic displacement field $(\vec{u})$. The system of equations thus becomes highly nonlinear.

\section{Solution of the Problem}

To solve the above problem, we apply the Fourier integral transform in the following form:

$$
\begin{aligned}
& n_{1}(x, z, t) \\
& \quad=\frac{1}{2 \pi} \iint_{-\infty}^{\infty} \tilde{n}(q, z, \omega) \exp [-i(q x+\omega t)] d q d \omega, \\
& \{u, v\}(x, z, t) \\
& \quad=\frac{1}{2 \pi} \iint_{-\infty}^{\infty}\{\tilde{u}, \tilde{v}\}(q, z, \omega) \exp [-i(q x+\omega t)] d q d \omega .
\end{aligned}
$$

Substitution of this into (9) gives

$$
\begin{aligned}
& \frac{\partial \widetilde{\sigma}_{z x}}{\partial z}-i q \widetilde{\sigma}_{x x}+\rho \omega^{2} \widetilde{u}=0 \\
& \frac{\partial \widetilde{\sigma}_{z z}}{\partial z}-i q \widetilde{\sigma}_{x z}+\rho \omega^{2} \widetilde{v}=0
\end{aligned}
$$

$$
\begin{aligned}
\frac{\partial^{2} \widetilde{n}}{\partial z^{2}} & -\left(q^{2}+\frac{i \omega \tau+1}{l^{2}}\right) \widetilde{n} \\
& =\frac{n_{0} \beta}{k_{B} T}\left(-i q^{3} \widetilde{u}+q^{2} \frac{\partial^{2} \widetilde{v}}{\partial z^{2}}+i q \frac{\partial^{2} \widetilde{u}}{\partial z^{2}}-\frac{\partial^{3} \widetilde{v}}{\partial z^{3}}\right),
\end{aligned}
$$

where

$$
\begin{aligned}
& \widetilde{\sigma}_{x x}=-i q(\lambda+2 \mu) \tilde{u}+\lambda \frac{\partial \widetilde{v}}{\partial z}-\beta \widetilde{n} \\
& \widetilde{\sigma}_{z z}=(\lambda+2 \mu) \frac{\partial \widetilde{v}}{\partial z}-i q \lambda \tilde{u}-\beta \widetilde{n} \\
& \widetilde{\sigma}_{x z}=\mu\left(\frac{\partial \tilde{u}}{\partial z}-i q \widetilde{v}\right),
\end{aligned}
$$

where $\omega$ is the frequency of wave propagation, $q$ is the wave number, and $l=\sqrt{D \tau}$; the phase velocity is given by $c=\omega_{r} / q$ and attenuation constant by $\Gamma=-\omega_{i}$, where $\omega_{r}=\operatorname{Re}(\omega)$ and $\omega_{i}=\operatorname{Im}(\omega)$ mean, respectively, the real and imaginary parts of $\omega . \lambda=\lambda(q)=\lambda_{0}\left(1-g^{2} q^{2}\right)$ and $\mu=\mu(q)=\mu_{0}\left(1-g^{2} q^{2}\right)$ are the nonlocal elastic moduli; $\beta=\beta(q)=\beta_{0}\left(1-h^{2} q^{2}\right)$ is the nonlocal constant characterizing lattice deformation due to atomic defects; $\widetilde{u}(q, z), \widetilde{v}(q, z)$, and $\widetilde{n}(q, z)$ are unknown functions (amplitude functions).

The general solutions to (13) are

$$
\begin{aligned}
\widetilde{u}=a_{1} \exp \left(-\eta_{1} z\right)+a_{2} \exp \left(-\eta_{2} z\right)+a_{3} \exp \left(-\eta_{3} z\right) \\
\widetilde{v}=-\frac{i q}{\eta_{1}} a_{1} \exp \left(-\eta_{1} z\right)+m_{2} a_{2} \exp \left(-\eta_{2} z\right) \\
+m_{3} a_{3} \exp \left(-\eta_{3} z\right) \\
\tilde{n}=d_{2} a_{2} \exp \left(-\eta_{2} z\right)+d_{3} a_{3} \exp \left(-\eta_{3} z\right)
\end{aligned}
$$

where

$$
\eta_{1}^{2}=q^{2}-\frac{\rho \omega^{2}}{\mu} .
$$

$\eta_{2}^{2}$ and $\eta_{3}^{2}$ are the roots of the equation

$$
\begin{aligned}
& (1-\delta) \eta^{4}-\eta^{2}\left[2 q^{2}(1-\delta)-\rho \frac{\omega^{2}}{\lambda+2 \mu}+\frac{i \omega \tau+1}{l^{2}}\right] \\
& +\left\{\left(q^{2}-\rho \frac{\omega^{2}}{\lambda+2 \mu}\right)\left(\frac{i \omega \tau+1}{l^{2}}+q^{2}\right)-\delta q^{4}\right\}=0,
\end{aligned}
$$

where $a_{i}, i=1,2,3$ are arbitrary constants, $\delta=n_{0} \beta^{2} /(\lambda+$ $2 \mu) k_{B} T$ is the coupling constant of nonlocal defect-strain interaction, and

$$
m_{j}=\frac{\eta_{j}}{i q}, \quad d_{j}=\frac{\lambda+2 \mu}{\beta i q}\left(\eta_{1}^{2}-\eta_{j}^{2}\right), \quad j=2,3 .
$$


Then $\eta_{2}^{2}$ and $\eta_{3}^{2}$ are defined from (17) as follows:

$$
\begin{aligned}
\eta_{2}^{2}+\eta_{3}^{2}= & {\left[2 q^{2}(1-\delta)-\rho \frac{\omega^{2}}{\lambda+2 \mu}+\frac{i \omega \tau+1}{l^{2}}\right] } \\
& \times(1-\delta)^{-1} \\
\eta_{2}^{2} \eta_{3}^{2}= & {\left[\left(q^{2}-\rho \frac{\omega^{2}}{\lambda+2 \mu}\right)\left(q^{2}+\frac{i \omega \tau+1}{l^{2}}\right)-\delta q^{4}\right] } \\
& \times(1-\delta)^{-1} .
\end{aligned}
$$
(8):

Stress components are obtained by substituting (15) into

$$
\begin{aligned}
\tilde{\sigma}_{x x}(q, z)= & -2 i q(\lambda+\mu) a_{1} \exp \left(-\eta_{1} z\right) \\
& +\left(-i q(\lambda+2 \mu)+\lambda \eta_{2} m_{2}+\beta d_{2}\right) a_{2} \exp \left(-\eta_{2} z\right) \\
& +\left(-i q(\lambda+2 \mu)+\lambda \eta_{3} m_{3}+\beta d_{3}\right) a_{3} \exp \left(-\eta_{3} z\right), \\
\tilde{\sigma}_{z z}(q, z)= & 2 i q \mu a_{1} \exp \left(-\eta_{1} z\right) \\
& +\left(i q \lambda-(\lambda+2 \mu) \eta_{2} m_{2}+\beta d_{2}\right) a_{2} \exp \left(-\eta_{2} z\right) \\
& +\left(-i q \lambda-(\lambda+2 \mu) \eta_{3} m_{3}+\beta d_{3}\right) a_{3} \exp \left(-\eta_{3} z\right), \\
\widetilde{\sigma}_{x z}(q, z)= & \eta_{1}\left(1+\frac{q^{2}}{\eta_{1}^{2}}\right) a_{1} \exp \left(-\eta_{1} z\right) \\
& +\left(\eta_{2}+i q m_{2}\right) a_{2} \exp \left(-\eta_{2} z\right) \\
& +\left(\eta_{3}+i q m_{3}\right) a_{3} \exp \left(-\eta_{3} z\right) .
\end{aligned}
$$

\section{Dispersion Equations of the Wave in an Infinitive Medium}

In this section as a special case we consider the generation of plane harmonic structures in unbounded nonlocal medium. Setting $\eta_{j}=0, j=1,2,3$ in (16) and (17) we obtain

$$
\begin{gathered}
\rho \omega^{2}=\mu(q) q^{2} \\
\left(q^{2}+\frac{i \omega \tau+1}{l^{2}}\right)\left[q^{2}-\omega^{2} \frac{\rho}{\lambda(q)+2 \mu(q)}\right]-\delta(q) q^{4}=0 .
\end{gathered}
$$

Suppose now that one ignores the influence of the nonlocal properties of the medium and sets $\lambda(q)=\lambda_{0}=$ const, $\mu(q)=\mu_{0}=$ const, and $\delta(q)=\delta_{0}=$ const. In this case (21) and (22) reduce to frequency equations

$$
\begin{gathered}
\omega^{2}=c_{T 0}^{2} q^{2} \\
\left(q^{2}-\omega^{2} c_{L 0}^{-2}\right)\left[q^{2}+(i \omega \tau+1) l^{-2}\right]-\delta_{0} q^{4}=0
\end{gathered}
$$

$\left(c_{L 0}=\left[\left(2 \mu_{0}+\lambda_{0}\right) / \rho\right]^{1 / 2}\right.$ and $c_{T 0}=\left(\mu_{0} / \rho\right)^{1 / 2}$ are the classical velocities of the longitudinal and shear waves, resp.), coinciding with the equations derived in the conventional local theory (see, e.g., [8]). In the nonlocal case, the frequency equation has, of course, a more complex structure.

To explore and delineate the strain and defect generation effects, we will seek solutions of (22) for small values of $\delta$. For $\delta=0,(22)$ admits the following solutions $\omega_{1,2}^{(0)}= \pm c_{L} q$ (acoustical mode) and $\omega_{3}^{(0)}=i \tau^{-1}\left(l^{2} q^{2}+1\right)$ (diffusion mode). Now, for small $\delta \ll 1$, we may write

$$
\begin{aligned}
& \omega_{1,2}=\omega_{1,2}^{(0)}+\delta \omega_{1,2}^{(1)}+\delta^{2} \omega_{1,2}^{(2)}+\cdots \\
& \omega_{3}=\omega_{3}^{(0)}+\delta \omega_{3}^{(1)}+\delta^{2} \omega_{3}^{(2)}+\cdots
\end{aligned}
$$

Substituting (24) into (22), and, equating the coefficients of like powers of $\delta$, we finally arrive to

$$
\begin{gathered}
\omega_{1,2}=\omega_{1,2}^{(0)}-\frac{\delta}{2} \frac{\omega_{1,2}^{(0)} q^{2} l^{2}\left(q^{2} l^{2}+1-i \omega_{1,2}^{(0)} \tau\right)}{\left(q^{2} l^{2}+1\right)^{2}+\left(\omega_{1,2}^{(0)} \tau\right)^{2}}+O\left(\delta^{2}\right), \\
\omega_{3}=\omega_{3}^{(0)}-\frac{i \delta l^{2} c_{L}^{2} q^{4} \tau}{c_{L}^{2} q^{2} \tau^{2}+\left(q^{2} l^{2}+1\right)^{2}}+O\left(\delta^{2}\right) .
\end{gathered}
$$

From (25) we obtain

$$
\begin{gathered}
\operatorname{Re}\left(\omega_{1,2}\right)=\omega_{1,2}^{(0)}\left[1-\frac{\delta}{2} \frac{q^{2} l^{2}\left(q^{2} l^{2}+1\right)}{\left(q^{2} l^{2}+1\right)^{2}+\left(c_{L} q \tau\right)^{2}}\right], \\
\operatorname{Im}\left(\omega_{1,2}\right)=\frac{\delta \tau}{2} \frac{c_{L}^{2} q^{4} l^{2}}{\left(q^{2} l^{2}+1\right)^{2}+\left(c_{L} q \tau\right)^{2}} .
\end{gathered}
$$

If $c_{L} q \gg \tau^{-1}\left(l^{2} q^{2}+1\right)$ and the viscosity is taken into account [by adding in (9) the terms $\eta_{T} \Delta \dot{\vec{u}}$ and $\eta_{L} \nabla$ (div $\left.\dot{\vec{u}}\right)$, where $\eta_{T}=\eta ; \eta_{L}=4 \eta / 3+\zeta ; \eta$ and $\zeta$ are the first and second viscosity coefficients] dispersion (28) describes attenuation of the amplitude of acoustic waves

$$
\operatorname{Im}\left(\omega_{1,2}\right)=\frac{1}{2 \rho} \eta q^{2}+\frac{\delta}{2 \tau}\left(l^{2} q^{2}+1\right)
$$

For the frequency spectrum of acoustic wave we have

$$
\operatorname{Re}\left(\omega_{1,2}\right)=\omega_{1,2}^{(0)}\left[1-\frac{\delta}{2 \tau} \frac{\left(l^{2} q^{2}+1\right)}{c_{L} q}\right] \approx \omega_{1,2}^{(0)}
$$

Note that the frequency of acoustic waves is hardly changed. But the additional contribution to attenuation coefficient of waves arises. As attenuation decrement of acoustic waves is proportional to $q^{2}$, according to (29), short waves fade fast.

$$
\begin{aligned}
& \text { If } c_{L} q \ll \tau^{-1}\left(l^{2} q^{2}+1\right) \\
& \operatorname{Re}\left(\omega_{1,2}\right)=\omega_{1,2}^{(0)}\left(1-\frac{\delta q^{2} l^{2}}{2\left(q^{2} l^{2}+1\right)}\right), \\
& \operatorname{Im}\left(\omega_{1,2}\right)=\frac{1}{2 \rho} \eta q^{2}+\frac{\delta \tau}{2} \frac{c_{L}^{2} q^{4} l^{2}}{\left(q^{2} l^{2}+1\right)^{2}} .
\end{aligned}
$$

In this case there is a softening of frequencies of acoustic waves (instability of frequencies $\operatorname{Re}\left(\omega_{1,2}\right) \rightarrow 0, \operatorname{Im}\left(\omega_{1,2}\right)>0$ ) 
and this is related to taking into account the generation of atomic defects. It is necessary to notice that reduction of frequency occurs not up to zero but up to value $\omega_{\eta}=$ $\eta q^{2} / 2 \rho \ll c_{L} q$. Equation (32) describes attenuation of the amplitudes of acoustic waves.

At $\omega_{i} \ll c_{L} q$ from (22) we obtain dispersion relation (growth rate) $\Gamma=-\operatorname{Im}(\omega)$ of the volume concentrationelastic instability:

$$
\Gamma \tau=l^{2} q^{2}(\delta-1)-1
$$

or

$$
\Gamma=D q^{2}\left[\delta_{0} \frac{\left(1-g^{2} q^{2}\right)^{2}}{1-h^{2} q^{2}}-1\right]-\frac{1}{\tau},
$$

where $\delta_{0}=n_{0} \beta_{0}^{2} /\left(\lambda_{0}+2 \mu_{0}\right) k_{B} T$.

Let us introduce the dimensionless growth rate $\widetilde{\Gamma}=$ $\Gamma g^{2} / D$, the wave number $\tilde{q}=q g$, the life-time of defects $\tilde{\tau}=\tau D / g^{2}$, and parameters $\gamma=g^{2} / h^{2}$. Dependence (34) in dimensionless variables takes the form

$$
\widetilde{\Gamma}=\frac{\widetilde{q}^{2}}{1-\widetilde{q}^{2}}\left(\widetilde{q}^{2}+\delta_{0}\left(1-\gamma \widetilde{q}^{2}\right)^{2}-1\right)-\frac{1}{\widetilde{\tau}} .
$$

We consider two situations that differ from each other by the relation $(\gamma)$ of the squares of the characteristic lengths $g$ and $h$. Figure 1 demonstrates the graphs of the growth rate calculated using (35) for two values of the ratio parameter $\gamma=8$ and 10 , respectively. The following typical values of parameters were used in the calculations: $h=0.6 \mathrm{~nm}$, $\delta_{0}=10$, and $D=4 \times 10^{-11} \mathrm{~cm}^{2} \mathrm{~s}^{-1}$. Comparison of the plots presented in Figure 1 shows that the dependences of the growth rates of the wave number for the two cases qualitatively differ from each other. It follows from Figure 1 that the growth rate exhibits a single maximum at $q_{m}=$ $3 \times 10^{6}$ for small values of $\gamma$ and an additional maximum in the short-wavelength region at $q>6 \times 10^{6} \mathrm{~cm}^{-1}$. Therefore, depending on the relation between the characteristic lengths $g$ and $h$, elastic-concentration structures have one or two maximum growth rates.

Figure 2 shows the dependences $\widetilde{\Gamma}(\widetilde{q})$ of the increment on the wave number for two values of the control parameter $\delta_{0}=5$ and 15 when the ratio parameter is $\gamma=8$. It follows from this figure that the growth rate has a single maximum not far above the threshold. However, at sufficiently high concentrations of the defects exceeding the threshold appears an additional maximum in the short-wavelength range. Therefore, as in the previous case, two gratings have the maximum growth rates.

The analytical expression for the maximum of the growth rates can be obtained provided that the nonlocal parameters are of the same order in (34) (i.e., $g \approx h$ ). Then, using (34), we have

$$
\Gamma(q)=D q^{2}\left[\frac{G}{G_{c}}\left(1-g^{2} q^{2}\right)-1\right]-\frac{1}{\tau},
$$

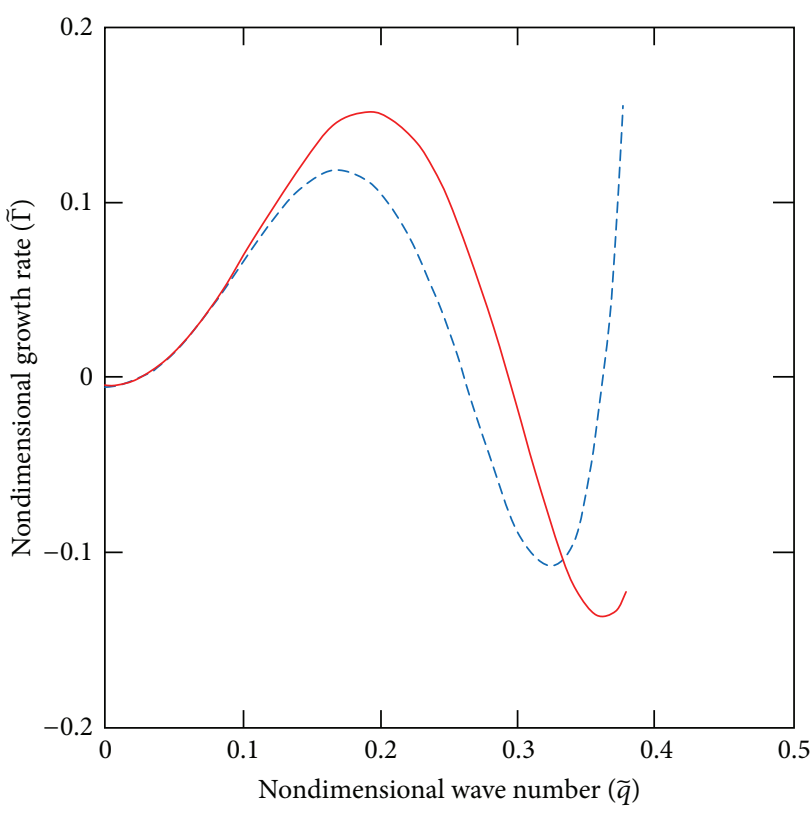

FIgURE 1: Dependences of the growth rates on the wave number for two values of the ratio parameter $\gamma: 8$ (solid line); 10 (dot line). Control parameter: $\delta_{0}=10$.

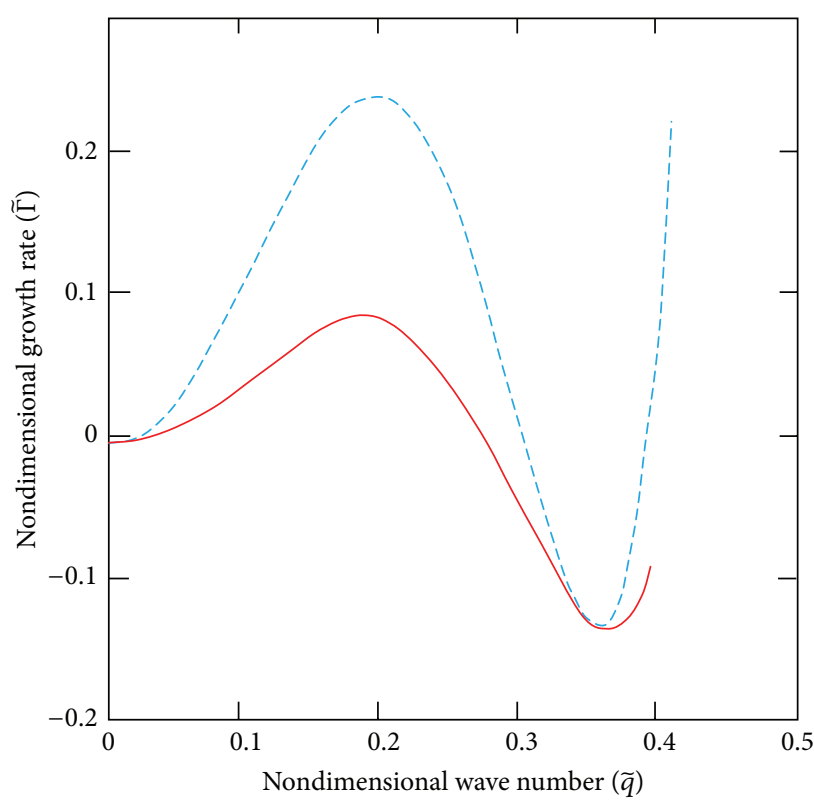

Figure 2: Dependences of the growth rate on the wave number for two values of the control parameter $\delta_{0}: 5$ (solid line), 15 (dot line). Ratio parameter: $\gamma=8.0$.

where

$$
G_{c}=k_{B} T \rho c_{L}^{2}\left(\tau \beta_{0}^{2}\right)^{-1}
$$

is the critical value of the control parameter (defect generation rate). So, putting values into (37) for molybdenum: $\beta_{0}=$ $20 \mathrm{eV}, \rho c_{L}^{2}=10^{12} \mathrm{erg} \mathrm{cm}^{-3}, T=10^{3} \mathrm{~K}$, and $\tau=10^{-3} \mathrm{~s}$, one can get the estimate $G_{c}=10^{24} \mathrm{~s}^{-1} \mathrm{~cm}^{-3}$. 
The dispersion curve $\Gamma(q)$ has a maximum $\Gamma_{m}$ at $q=$ $q_{m}$, which determines the spatial scale $d_{\text {latt }}=2 \pi / q_{m}$ of the dominant CS structures:

$$
d_{\text {latt }}=\sqrt{8} \pi g \sqrt{\frac{G}{G-G_{c}}} .
$$

The maximum growth rate is

$$
\Gamma_{m}=\frac{D}{4 g^{2}} \frac{\left(1-G_{c} / G\right)^{2}}{G / G_{c}}-\tau^{-1} .
$$

It is seen from (39) that the development of the elasticconcentration instability with the self-organization of CS structures in the volume of the solids occurs in a threshold manner when the defect generation rate exceeds the critical value: $G>G_{c}$. At the elastic-concentration instability threshold $\left(G \rightarrow G_{c}\right.$ ), the period tends to infinity $d_{\text {latt }} \rightarrow$ $\infty$. At high values of generation rates $G \gg G_{c}$, the period asymptotically tends to its minimal value $d_{\text {latt }}=\sqrt{8} \pi g$.

Near the threshold $G \rightarrow G_{c}$ the time of formation of the CS structures $\Gamma_{m}^{-1} \rightarrow \infty$ that is critical slowing down takes place, which is characteristic of the phase transitions of the second kind.

The critical value of the generation rate derived here is directly proportional to recombination rate $\left(\tau^{-1}\right)$ and inversely proportional to deformational potentials $\left(\beta_{0}\right)$ of defects. Putting $\tau=\tau_{0} \exp \left(w_{m} / k_{B} T\right)$ into (37) for the generation rate, we get

$$
G_{c}(T)=\frac{\rho c_{L}^{2} k_{B} T}{\tau_{0} \beta_{0}^{2}} \exp \left(-\frac{w_{m}}{k_{B} T}\right) .
$$

One notes that this dependence is very rapid, which is largely due to variation of the defect relaxation time with temperature.

From (38), it follows that the period of the volume ordered CS structures is proportional to the characteristic length $h$ of the defect interaction with the crystal-lattice atoms, which lies in the obtained nanometer range. As temperature is increased, the period grows for a particular value of $h$. For a particular value of $T$, the period decreases with an increasing generation rate $(G)$.

A periodic structure of vacancy clusters in the bulk of a solid irradiated with nitrogen ions was first observed in 1971 by Evans in pure molybdenum [24]. The medium temperature was $T=1040^{\circ} \mathrm{K}$. Subsequently, the same effect had been observed in other metals such as aluminum, tungsten, nickel, and niobium $[25,26]$. Vacancy cluster sizes in the lattice were 2-4 $\mathrm{nm}$, and values of the lattice parameter change in the range of (20-60) nm. At $g=1.5 \mathrm{~nm}$ (for $G=1.1 G_{c}$ ) from (38) we obtain an estimate for the period of the volume structure $d_{\text {latt }}=44 \mathrm{~nm}$. This is in qualitative agreement with the abovementioned experimentally observed interval.

Note that the linear theory considered in this section describes early stage of the development of an elasticconcentration instability only. However, the nature of generated ordered volume structures (due to an instability) and the amplitudes of these structures as functions on material and irradiation conditions can only be determined by considering the influence of elastic nonlinear effects in the model.

\section{Dispersion Equations of the Surface Concentration-Strain Structures}

Substituting expressions (20) into boundary conditions (10) and taking into account expressions (18), we find a set of three linear algebraic equations:

$$
\begin{gathered}
2 q^{2} a_{1}+\left(\eta_{1}^{2}+q^{2}\right) a_{2}-\left(\eta_{1}^{2}+q^{2}\right) a_{3}=0 \\
\left(\eta_{1}^{2}+q^{2}\right) a_{1}+2 \eta_{2} \eta_{1} a_{2}+2 \eta_{3} \eta_{1} a_{3}=0 \\
\eta_{2}\left(\eta_{2}^{2}-q^{2}+\frac{\rho \omega^{2}}{\lambda+2 \mu}\right) a_{2}+\eta_{3}\left(\eta_{3}^{2}-q^{2}+\frac{\rho \omega^{2}}{\lambda+2 \mu}\right) a_{3}=0 .
\end{gathered}
$$

The condition of existence of a nontrivial solution of this system yields

$$
\left(\eta_{1}^{2}+q^{2}\right)^{2}=\frac{4 \eta_{1} \eta_{2} \eta_{3} q^{2}\left(\eta_{2}+\eta_{3}\right)}{\eta_{2}^{2}+\eta_{3}^{2}+\eta_{2} \eta_{3}+\rho \omega^{2}(\lambda+2 \mu)^{-1}-q^{2}},
$$

where $\eta_{1}, \eta_{2}$, and $\eta_{3}$ are given by (16) and (19).

Equation (42) shows dispersive character of the Rayleigh waves propagating in an elastic half-space. This dispersive character of these waves propagation arises due to atomic defect generation in the medium with long-range interactions.

Note that the form of this dispersion equation is identical to the corresponding equation obtained in the context of concentration-elastic coupled theory, using classical constitutive equations of motion, though each of $\eta_{1}, \eta_{2}$, and $\eta_{3}$ represents different expressions for classical elasticity and nonlocal elasticity.

Equation (21) is generalized form of the classical local Raleigh equations. The assumption of vanishing defect generation in this equation gives us exactly the same results as those obtained by Nowiński [17] for solids with long-range interactions; that is,

$$
\begin{gathered}
4 q^{2} \bar{\eta}_{1} \bar{\eta}_{2}-\left(\bar{\eta}_{1}^{2}+q^{2}\right)^{2}=0 \\
\bar{\eta}_{1}^{2}=q^{2}-\frac{\rho \omega^{2}}{\mu}, \quad \bar{\eta}_{2}^{2}=q^{2}-\frac{\rho \omega^{2}}{(\lambda+2 \mu)} .
\end{gathered}
$$

\section{Solution of Dispersion Equation}

A general discussion of (42) presents considerable difficulties. The structure of (42) is identical to the structure of the dispersion equation for DEI considered in [8]. This equation can be readily solved numerically or graphically, but in some cases of interest it can also be solved analytically. We shall now consider the results for some limiting cases. We can rewrite (42) as

$$
\left(1+\frac{\eta_{1}^{2}}{q^{2}}\right)^{2}=\frac{4 \eta_{1} B(A+2 B)^{1 / 2}}{q^{2}\left(A+B-\bar{\eta}_{2}^{2}\right)},
$$

where $A=\eta_{2}^{2}+\eta_{3}^{2}$ and $B=\eta_{2} \eta_{3}$. 
Equation (45) has solutions describing qualitatively different types of instability: (1) instability of frequencies of surface acoustic waves and (2) generation of ordered surface (static) concentration-strain structures.

If the coupling term is small $(\delta \ll 1)$ the expressions that appear in the dispersion equation (25), to a first approximation, then read

$$
\begin{gathered}
B=\bar{\eta}_{2} \bar{\eta}_{3}\left(1+\delta \frac{\left(\bar{\eta}_{2}^{2} \bar{\eta}_{3}^{2}-q^{4}\right)}{2 \bar{\eta}_{2}^{2} \bar{\eta}_{3}^{2}}\right), \\
A=\bar{\eta}_{2}^{2}+\bar{\eta}_{3}^{2}+\delta\left(\bar{\eta}_{2}^{2}+\bar{\eta}_{3}^{2}-2 q^{2}\right), \quad \bar{\eta}_{3}^{2}=q^{2}+\frac{(i \omega \tau+1)}{l^{2}} .
\end{gathered}
$$

Then we may express the dispersion equation in the following form:

$$
R(\omega, q)-\delta Z(\omega, q)=0
$$

Here

$$
\begin{gathered}
R(\omega, q)=\left(\bar{\eta}_{1}^{2}+q^{2}\right)^{2}-4 q^{2} \bar{\eta}_{1} \bar{\eta}_{2}, \\
Z(\omega, q)=\frac{2 q^{2} \bar{\eta}_{1}\left(2 \bar{\eta}_{2}+\bar{\eta}_{3}\right)\left(\bar{\eta}_{2}^{2}-q^{2}\right)^{2}}{\bar{\eta}_{3} \bar{\eta}_{2}\left(\bar{\eta}_{2}+\bar{\eta}_{3}\right)^{2}} .
\end{gathered}
$$

For $\delta=0$ we obtain the nonlocal elastic solution given by Nowiński [17]. Introducing the dimensionless variable $\xi=$ $\left(\omega / \bar{c}_{1} q\right)^{2}$ we may write the dispersion equation (47) in the following form:

$$
R(\xi)-\delta Z(\xi)=0 .
$$

Now we may consider that $\varepsilon$ is the increment of the value of $\xi$ due to $\delta \neq 0$. Then (49) can be rewritten in the form of

$$
R\left(\xi_{R}+\varepsilon\right)-Z\left(\xi_{R}+\varepsilon\right) \delta=0,
$$

where $\xi_{R}$ is a root of the equation $R\left(\xi_{R}\right)=0$.

Assuming that $\varepsilon \ll 1$, we may expand both sides of (50) into a Taylor series in the vicinity of the point $\xi=\xi_{R}$. Retaining only the first two terms, we obtain

$$
\varepsilon\left(\xi_{R}, \delta\right)=-\frac{Z\left(\xi_{R}\right)}{\left.(\partial R / \partial \xi)\right|_{\xi=\xi_{R}}} \delta=\varepsilon_{r}+i \varepsilon_{i},
$$

where $\varepsilon_{r}=\operatorname{Re}(\varepsilon)$ and $\varepsilon_{i}=\operatorname{Im}(\varepsilon)$.

The real part of $\varepsilon$ characterizes the change of the phase velocity and its imaginary part $\left(\varepsilon_{i}\right)$ defines the attenuation constant. We have

$$
\xi=\frac{1}{\bar{c}_{1}^{2}}\left(\frac{\omega}{q}\right)^{2}=\xi_{R}+\varepsilon, \quad \omega=q \bar{c}_{1}\left(\varepsilon_{r}+\xi_{R}+i \varepsilon_{i}\right)^{1 / 2} .
$$

This equation gives the following expressions:

$$
\begin{gathered}
\omega_{r}=\bar{c}_{1} q \sqrt{\xi_{R}}\left(\frac{1-\delta\left|Z_{r}\right|}{2 \xi_{R} M}\right), \\
\omega_{i}=\frac{\bar{c}_{1} q \delta Z_{i}}{\xi_{R} M},
\end{gathered}
$$

where $Z_{r}=\operatorname{Re}(Z), Z_{i}=\operatorname{Im}(Z)$, and $M=\left.(\partial R / \partial \xi)\right|_{\xi=\xi_{R}}$.
Then for the phase velocity we have

$$
c=\bar{c}_{1} \sqrt{\xi_{R}}\left(1-\frac{\delta\left|Z_{r}\right|}{2 \xi_{R} M}\right) .
$$

Since $\delta>0$, in this case there is a softening of frequencies of acoustic waves (instability of frequencies $\omega_{r} \rightarrow 0$ and $\omega_{i}>0$ ) and this is related to taking into account the generation of atomic defects. Equation (53b) describes attenuation of the amplitudes of acoustic waves.

Expanding $\eta_{1}$ and $\eta_{2}$ in powers of the small parameter $\omega_{i} / c_{T \cdot L} \ll 1\left(\omega_{r}=0\right)$, we obtain from (21) the expression for the growth rate $\Gamma=-\omega_{i}$ of the surface periodical static structures in the form

$$
\Gamma=D q^{2}\left[\frac{\delta}{\left(1-\zeta^{-1}\right)} \frac{\left(1-h^{2} q^{2}\right)^{2}}{\left(1-g^{2} q^{2}\right)}-1\right]-\tau^{-1},
$$

where $\zeta=c_{L}^{2} c_{T}^{-2}$.

Introducing the dimensionless growth rate $\widetilde{\Gamma}=\Gamma g^{2} / D$, the wave number $\tilde{q}=q g$, the life-time of defects $\tilde{\tau}=\tau D / g^{2}$, and parameters $\phi=\delta /\left(1-\zeta^{-1}\right), \gamma=g^{2} / h^{2}$, dependence (34) can be represented as

$$
\widetilde{\Gamma}=\frac{\widetilde{q}^{2}}{1-\widetilde{q}^{2}}\left(\widetilde{q}^{2}+\phi\left(1-\gamma \widetilde{q}^{2}\right)^{2}-1\right)-\widetilde{\tau}^{-1} .
$$

The analysis shows that the growth rate exhibits a single maximum at $q=q_{m}$ for small values of ratio parameter $(\gamma)$ and an additional maximum $\left(q_{c}\right)$ in the short-wavelength region for large values of $\gamma$. Thus depending on the relation between the characteristic lengths $g$ and $h$, elasticconcentration structures can have one or two (with $q=q_{m}$ and $q=q_{c}$ ) maximum growth rates.

Besides the growth rate has a single maximum not far above the threshold. However, at sufficiently high concentrations of the defects exceeding the threshold appears an additional maximum in the short-wavelength range. Therefore, as in the previous case, two gratings have the maximum growth rates.

The obvious expression for the maximum of the growth rates can be obtained if the scale coefficients $(g, h)$ are of the same order in (34):

$$
\widetilde{\Gamma}=\widetilde{q}^{2}\left[\phi\left(1-\widetilde{q}^{2}\right)-1\right]-\widetilde{\tau}^{-1} .
$$

The growth rate $\widetilde{\Gamma}(\widetilde{q})$ reaches maximum $\Gamma_{m}$ at

$$
\widetilde{q}=\widetilde{q}_{m}=\frac{1}{\sqrt{2}} \sqrt{1-\frac{G_{c}^{(s)}}{G}},
$$

where

$$
G>G_{c}^{(s)}=\frac{k_{B} T \rho c_{L}^{2}\left(1-c_{T}^{2} c_{L}^{-2}\right)}{\tau \beta_{0}^{2}}
$$

is the critical value of the control parameter, at exceeding of which the self-organization of surface-ordered CS structures becomes possible due to development of the elasticconcentration instability. As a result, the surface deformation 
lattice and the corresponding relief modulation with the period $d_{\text {latt }}^{(s)}$ grow in time on the surface, as well as the defect concentration lattice (defect accumulations at the extrema of the surface relief).

The spatial scale of surface SC structures with wave number $q_{m}$ is given by

$$
d_{\text {latt }}^{(s)}=\frac{2 \pi}{q_{m}}=\sqrt{8} \pi g \sqrt{\frac{G}{G-G_{c}^{(s)}}} .
$$

The maximum growth rate is

$$
\Gamma_{m}=\frac{D}{4 g^{2}} \frac{G}{G_{c}^{(s)}}\left(1-\frac{G}{G_{c}^{(s)}}\right)^{2}-\tau^{-1} .
$$

Near the threshold $G \rightarrow G_{c}$ the time of formation of the CS structures $\Gamma_{m}^{-1} \rightarrow \infty$ that is critical slowing down takes place, which is characteristic of the phase transitions of the second kind.

The period of the ordered CS structures formed on the surface is proportional to the characteristic length $h$ of the nonlocal defect-atom interaction, which lies in the obtained nanometre range. As temperature is increased, the period grows for a particular value of $h$. For a particular value of $T$, the period decreases with an increasing generation rate $(G)$.

Thus at certain conditions concentration-elastic instabilities in a system of atomic defects in unbounded condensed media as well as on the free surface of a solid half-space can be developed. We have observed that if the pump parameter is above the critical value, due to concentrationelastic instability, coupled strain-defect periodic nanometer sized structures on the surface of solids arise. A mechanism on the development of the instability is due to the coupling between defect dynamics and the elastic field of the solids. Laser radiation (or, in general, a flux of particles) generates high concentrations of mobile atomic defects in the surface layer of the irradiated material. When a fluctuation harmonic of the elastic deformation field appears in a medium because of the generation of atomic defects, the strain-induced drift of atomic defects occurs. This is a consequence of nonlocal defect-strain interaction. The strain-induced flux of defects gives rise to periodic spatial-temporal fields of the defect concentration. The redistribution of defects creates forces proportional to their gradients. These forces lead in turn to additional growth of strain fluctuations. When a certain critical value of the defect density or rate of defect generation is exceeded, diffusion-elastic instabilities develop as a result of positive feedback, which result in the formation of ordered CS structures.

As an example let us consider the formation of periodic surface CS structures in laser-irradiated semiconductors (in particular, $C d T e$ ). To evaluate the concentration of generated lattice defects $\left(n_{0}\right)$, we consider here conditions when the duration of a laser pulse ( $\left.\tau_{\text {Las }}\right)$ exceeds the defect-relaxation time $(\tau)$. In this case the density of defects on the surface of the solid reaches a steady-state value

$$
n_{0}=G_{0} \tau=G_{0} \tau_{0} \exp \left(\frac{-w_{d 0}}{k_{B} T_{0}}\right),
$$

where

$$
w_{d 0}=w_{f 0}-w_{m 0}, \quad G_{0}=G\left(T_{0}\right)
$$

is the defect generation rate, $T_{0}$ is the steady-state value of the temperature field on the surface, and $G^{(0)}$ and $\tau_{0}$ are constants. If $I_{\text {Las }}=$ const (uniform irradiation) and the optical absorption length $\left(\alpha_{\text {Las }}^{-1}\right)$ is sufficiently less than heat diffusion length $l_{T}=\left(\chi \tau_{\text {Las }}\right)^{1 / 2}$, the maximum temperature rise at the substrate surface owing to the laser pulse action may be evaluated [22] as

$$
T_{0}=\frac{2(1-R) I_{0}}{\lambda_{T}} \sqrt{\frac{\chi \tau_{\text {Las }}}{\pi}},
$$

where $R$ is the reflectivity coefficient, $\lambda_{T}$ is the thermal conductivity coefficient, and $\chi$ is the thermal diffusion coefficient. So putting values into (64) for CdTe $\left(\lambda_{T}=\right.$ $0.05 \mathrm{~W} / \mathrm{cm}^{\circ} \mathrm{K}, \chi=3 \times 10^{-3} \mathrm{~cm}^{2} / \mathrm{s}, I_{0}=1.2 \times 10^{7} \mathrm{~W} / \mathrm{cm}^{2}$, pulse duration $2 \times 10^{-8} \mathrm{~s}$, and $R=0.4$ ) one can get $T_{0}=1.2 \times 10^{3}{ }^{\circ} \mathrm{K}$. Then, taking $\Omega_{d}=10^{-22} \mathrm{~cm}^{3}, K=5.10^{11} \mathrm{erg} / \mathrm{cm}^{3}, \zeta=2.44$, and $w_{d 0}=1 \mathrm{eV}$, a value of $2 \times 10^{19} 1 / \mathrm{cm}^{3}$ may be estimated for the critical density of defect concentration $\left(n_{c}=G_{c}^{(s)} \tau\right)$, which is several orders of magnitude less than the concentration of the host atoms and shows that this mechanism of the formation of ordered structures may be realized on practice. For the period $\left(d_{\text {latt }}\right)$ of the resultant surface CS structure we have an estimate of $71 \mathrm{~nm}$, which follows from expression (60) (for typical values of parameters $\rho=5.85 \mathrm{~g} / \mathrm{cm}^{3}, \beta_{0}=$ $30 \mathrm{eV}, c_{L}=2.4 \times 10^{5} \mathrm{~cm} / \mathrm{s}, G=1.1 G_{c}, 2 \pi g=15 \mathrm{~nm}$, $D=10^{-6} \mathrm{~cm}^{2} / \mathrm{s}$, and $\left.\tau=3 \times 10^{-9} \mathrm{~s}\right)$. The maximum growth increment of the instability is $\Gamma_{m}=6 \times 10^{8} \mathrm{~s}^{-1}$; the instability increment exceeds the reciprocal of the duration of a laser pulse acting on the surface of a solid $\left(\Gamma_{m} \tau_{\text {Las }}=12 \gg 1\right)$.

The appearance of periodic nanostructures of the surface relief was detected experimentally [27] when the surface of the $\mathrm{p}$-CdTe semiconductor crystal was irradiated by single pulses of the multimode ruby laser (pulse duration $\tau_{\text {Las }}=$ $2 \times 10^{-8}$ s) with a homogeneous distribution of intensity. The period of the relief structure (with lateral size of the nanoclusters $\propto(19-23) \mathrm{nm})$ was $74 \mathrm{~nm}$, which is in agreement with the predictions of our theory.

\section{Conclusions}

Based on the nonlocal elastic theory, which accounts for the small-scale effect by assuming the stress at a reference point as a function of the strain at every point in the body, the governing equations for the elastic displacement vector and defect density fields in an isotropic laser-excited solid have been derived. The defect-density dynamics are controlled by the generation and annihilation processes and also the strainstimulated transport of defects. We have provided exact formal solutions for the displacements and defect density fields in an elastic solid half-space. A linear analysis is used to obtain dispersion equations of surface waves through the solution of three coupled integropartial differential equations, by assuming that the surface of the solids is stressfree. A procedure for determining the phase velocity and 
the attenuation (amplification) factor has been discussed. The proposed analysis is applied for the special case of very short wavelengths. The phase velocity and attenuation constants of the waves get modified due to the defect subsystem of the solid. We have shown that the dispersion equations predict two types of the instability: (1) instability of frequencies of acoustic waves and (2) generation of ordered (surface or volume) structures.

We observed that the small-scale effect plays an important role in the dispersion behaviors for larger wave numbers. We have demonstrated that due to the nonlocal character of the defect-atom and atom-atom interactions the dispersion curves of the instability have two maxima sufficiently far above the instability threshold. When the regular surface relief is generated, two maxima of the growth rate must give rise to two scales of the surface relief. These two scales of the relief are the characteristic of the surface nanostructure self-organization upon both laser and ion-beam irradiation of semiconductors.

In the case of the laser molecular-beam epitaxy (MBE) and the laser-controlled deposition process of atoms on substrates, the extrema (minima or maxima) of the surface relief formed by the CS structure serve as the nucleation and growth centers of the nanoparticles. Similarly, in the processes of multipulse laser etching, the rapid material removal also takes place on the extremes of the CS field, where the removal rate takes maximum values. As a result, on the surface of substrate, size distribution functions of the nanoscale nonhomogeneities having two maxima can be formed. A transformation of the unimodal distribution to the bimodal (with two maxima) distribution upon a variation in the irradiation regime (control parameter) is typical of both the MBE and the laser-induced surface nanostructuring of metals and semiconductors. Formation of bimodal metal $\mathrm{Au})$ nanoparticle size distribution function upon the pulsed laser deposition was detected experimentally in [28].

The results obtained in this work are expected to be helpful in designing the nanostructures in small-scale physical devices.

\section{References}

[1] F. Mirzade, "Nonlinear strain waves interacting with laserinduced carries of the local disorder," in Laser Technologies of Materials Treatment, V. Y. Panchenko, Ed., pp. 220-277, Fizmatlit, Moscow, Russia, 2009, Russian.

[2] F. Mirzade, "Elastic wave propagation in a solid layer with laserinduced point defects," Journal of Applied Physics, vol. 110, no. 6, Article ID 064906, 2011.

[3] F. Mirzade, "A model for the propagation of strain solitary waves in solids with relaxing atomic defects," Journal of Applied Physics, vol. 103, no. 4, Article ID 044904, 2008.

[4] F. Mirzade, "Influence of atomic defect generation on the propagation of elastic waves in laser-excited solid layers," Physica B, vol. 406, no. 24, pp. 4644-4651, 2011.

[5] A. M. Kosevich, Physical Mechanics of Real Crystals, Nauka, Moscow, Russia, 1986.
[6] F. Mirzade, V. Y. Panchenko, and L. A. Shelepin, "Laser control of processes in solids," Uspechi Phyzicheskih Nauk, vol. 39, no. 1, pp. 3-30, 1996.

[7] F. Mirzade, "Concentration-elastic instabilities in a solid halfspace," Physica Status Solidi B, vol. 246, no. 7, pp. 1597-1603, 2009.

[8] F. Mirzade, "On diffusion-elastic instabilities in a solid halfspace," Physica B, vol. 406, no. 1, pp. 119-124, 2011.

[9] D. Walgraef and N. Ghoniem, "Modeling laser-induced deformation patterns: nonlinear effects and numerical analysis," Journal of Computer-Aided Materials Design, vol. 6, no. 2, pp. 323-335, 1999.

[10] F. Mirzade, "Finite-amplitude strain waves in laser-excited plates," Journal of Physics: Condensed Matter, vol. 20, no. 27, Article ID 275202, 2008.

[11] A. C. Eringen, Nonlocal Continuum Field Theories, Springer, New York, NY, USA, 2002.

[12] A. I. Kunin, Elastic Media with Microstructure, Springer, New York, NY, USA, 1982.

[13] A. C. Eringen, "On differential equations of nonlocal elasticity and solutions of screw dislocation and surface waves," Journal of Applied Physics, vol. 54, no. 9, pp. 4703-4710, 1983.

[14] J. N. Reddy, "Non-local nonlinear formulations for bending of classical and shear deformation theories of beams and plates," International Journal of Engineering Science, vol. 48, no. 11, pp. 1507-1518, 2010.

[15] A. Alibeigloo, "Free vibration analysis of nano-plate using three-dimensional theory of elasticity," Acta Mechanica, vol. 222, no. 1-2, pp. 149-159, 2011.

[16] B. Arash and Q. Wang, "A review on the application of nonlocal elastic models in modeling of carbon nanotubes and graphenes," Computational Materials Science, vol. 51, no. 1, pp. 303-313, 2012.

[17] J. L. Nowiński, "On non-local theory of wave propagation in elastic plates," ASME Journal of Applied Mechanics, vol. 51, no. 3, pp. 608-613, 1984.

[18] J. L. Nowinski, "On the propagation of thermoelastic waves in media with long-range cohesive forces," Journal of Thermal Stresses, vol. 10, no. 1, pp. 17-27, 1987.

[19] K. L. Pan and J. Fang, "Interaction energy of dislocation and point defect in bcc iron," Radiation Effects and Defects in Solids, vol. 139, no. 2, pp. 147-154, 1996.

[20] K. L. Pan, "An image force theorem for a screw dislocation near a crack in non-local elasticity," Journal of Physics D, vol. 27, no. 2, pp. 344-346, 1994.

[21] K.-L. Pan, "Interaction of a dislocation with a surface crack in nonlocal elasticity," International Journal of Fracture, vol. 69, no. 4, pp. 307-318, 1995.

[22] W. W. Duley, Laser Processing and Analysis of Materials, Plenum Press, New York, NY, USA, 1983.

[23] L. D. Landau and E. M. Lifshitz, Theory of Elasticity, Pergamon Press, Oxford, UK, 3rd edition, 1986.

[24] J. H. Evans, A. W. MacLean, A. A. Ismail, and D. Love, "Concentrations of plasma testosterone in normal men during sleep," Nature, vol. 229, pp. 261-262, 1971.

[25] V. K. Sikka and J. Moteff, "Damage in neutron-irradiated molybdenum," Journal of Nuclear Materials, vol. 54, no. 2, pp. 325345, 1974. 
[26] V. F. Zelensky, I. M. Nekludov, and L. S. Ozhigov, Some Problems in the Physics of Radiation Damage in Materials, Naukova Dumka, Kiev, Ukraine, 1979.

[27] A. Baidullaeva, A. B. Vlasenko, L. F. Cuzan et al., "Formation of nanosize structures on a surface of $p$-CdTe crystals due to the ruby laser single pulse irradiation," Semoconductors, vol. 39, pp. 1064-1067, 2005.

[28] J. Gonzalo, A. Perea, D. Babonneau et al., "Competing processes during the production of metal nanoparticles by pulsed laser deposition," Physical Review B, vol. 71, no. 12, Article ID 125420 , 8 pages, 2005. 

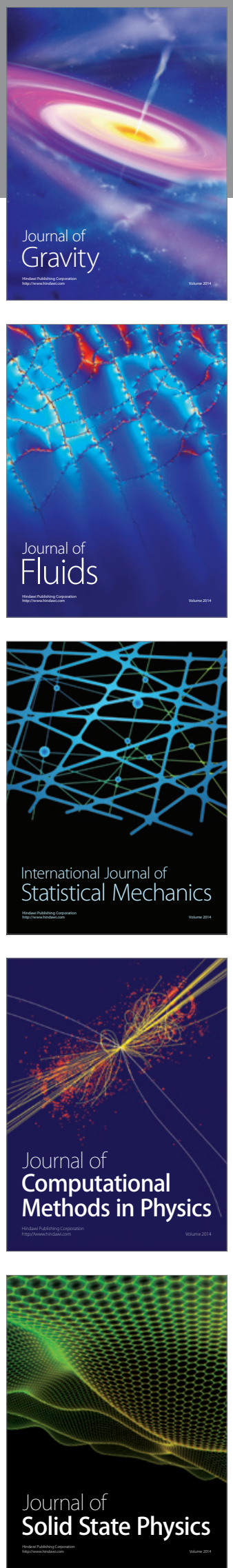

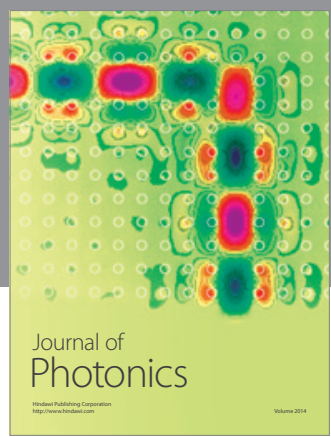

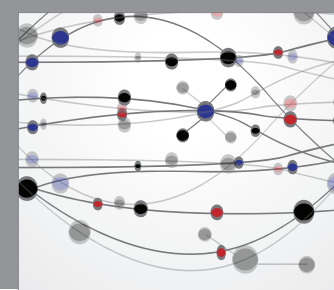

The Scientific World Journal

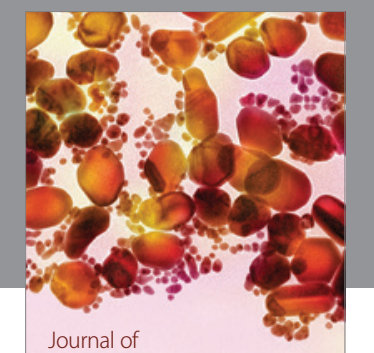

Soft Matter
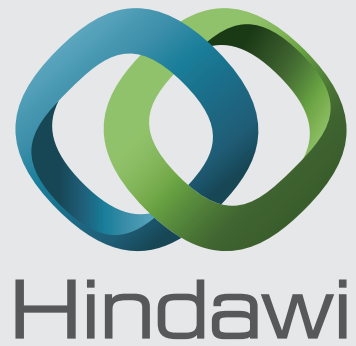

Submit your manuscripts at

http://www.hindawi.com
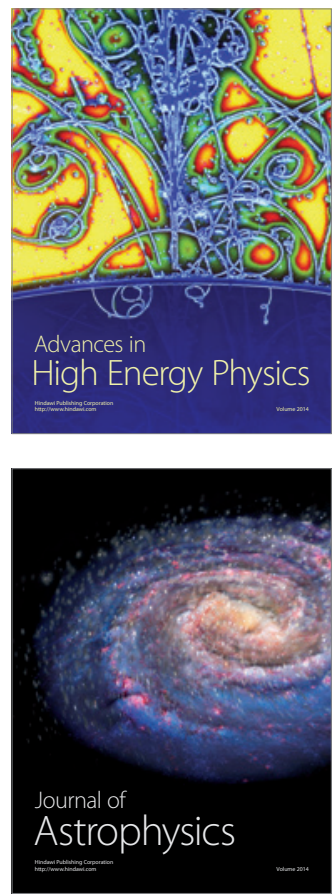
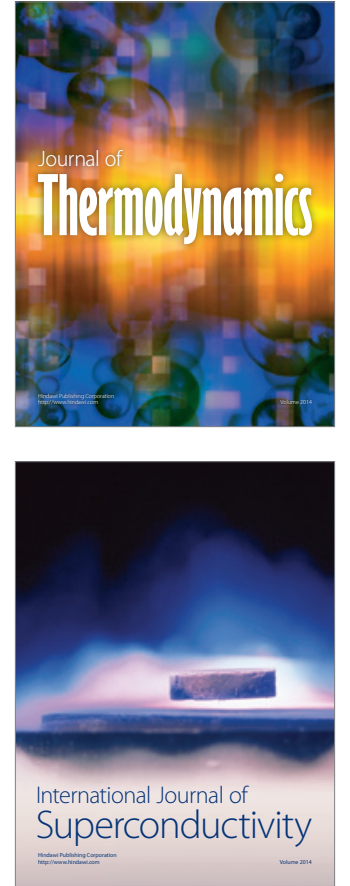
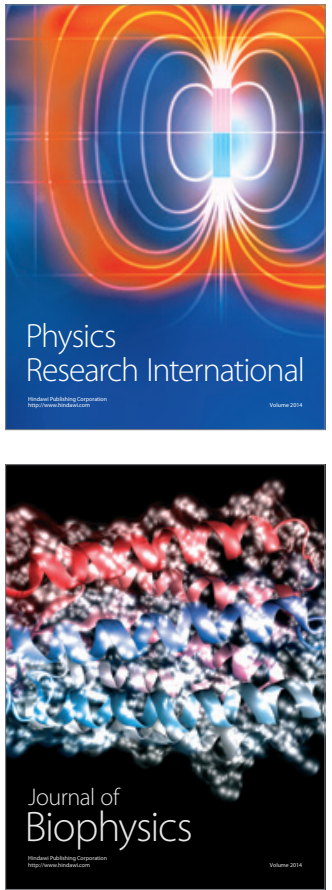
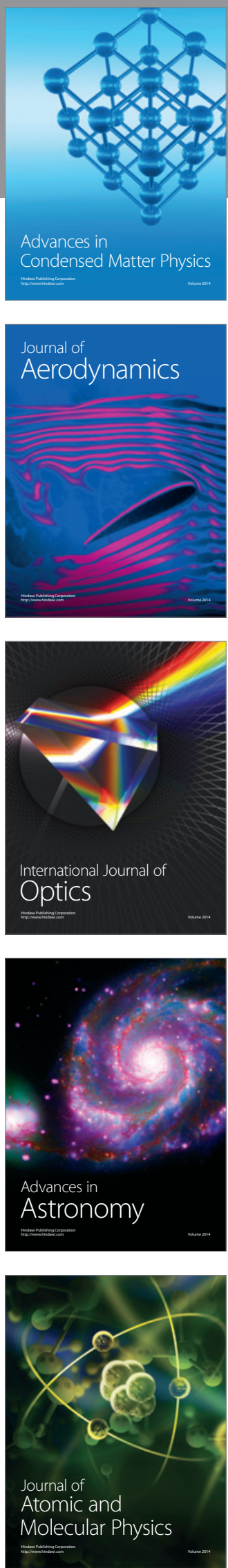